\title{
The Sensitivity Analysis of Bi-level Decision Problem Based on Modified Simplex Method
}

\author{
Yujie Tao \\ Department of Mathematics of Tonghua Normal University \\ Tonghua, China \\ taoyujie@126.com
}

\begin{abstract}
The bi-level decision making system involves two optimization decision problems. The target value of the upper level decision problem is implicitly determined by the solution of the lower problem. In This paper discusses the sensitivity analysis of bi-level linear programming while the value coefficients of the lower level decision contains parameter and studies the conditions of optimal solution while parameter continue (or perturbation) in definite direction. The result provides the decision basis for decision maker.)
\end{abstract}

Keywords- Bi-level decision, Sensitivity analysis, Parameter, Feasible set, Admissible se

\section{INTRODUCTION}

All manuscripts must be in English. These guidelines include complete descriptions of the fonts, spacing, and related information for producing your proceedings manuscripts. Please follow them and if you have any questions, direct them to the production editor in charge of your proceedings at Conference Publishing Services (CPS): Phone +1 (714) 821-8380 or Fax +1 (714) 761-1784.

References [1-2] of the paper are all research on when certain coefficient of upper or lower level objective function changes, if keeping the original optimal solution unchanging, what the maximum permissible variation range of the coefficient is? Reference [3] of the paper expounds when the upper objective function's coefficient includes function, analyzing the maximum variation range of parameter if keeping the optimal solution unchanging and analyzing the changing situation of optimal solution if exceeding the range.

On the basis of References [1-3] of the paper, utilizing simplex to discuss the sensitivity analysis of bi-level decision problem of lower level objective function to research the state of optimal solution when parameters continue (or perturb) in definite direction so as to calculate the relative changes of optimal solution by using the smallest supplement. Because of the solving ability of linear programming, the analysis of the paper only confine to the linear perturbation of parameters. In this continuous change process, the optimal solution is analyzed.

\section{PROPAEDEUTICS}

The basic model $\mathrm{M}$ of the bi-level decision problem that we are discussing is as follow:

$$
\begin{aligned}
& \max _{x} f_{1}(x, y)=a^{T} x+b^{T} y \text { (Where the solution of } y \text { is) } \\
& \qquad \max _{y} f_{2}(x, y) \\
& \text { s.t. } A x+B y \leq p
\end{aligned}
$$

Where

$a, x \in R^{n_{1}}, b, y \in R^{n_{2}}, p \in R^{m}, A \in R^{m \times n_{1}}, B \in R^{m \times n_{2}} \quad\left(n_{1}, n_{2}, m \quad\right.$ are dimensions of $x, y, p$ respectively, $R^{n}$ describes $\mathrm{n}$ dimensional space) and $f_{2}(x, y)$ is $y$,s linear function when $x$ is preset.

In the above models, if the cost coefficient of level objective function is the linear function of parameter, the following model $\mathrm{N}$ can be obtained, that is

$$
\begin{gathered}
\max _{x \quad 1} f(x, y) \\
\text { (Where the solution of }{ }^{y} \text { is) } \\
\max _{y} f_{2}(x, y)=a(t)^{T} x+b(t)^{T} y \\
\text { s.t. } A x+B y \leq p \\
x, y \geq 0
\end{gathered}
$$

Where $a(t), b(t)$ are linear functions of $t$, $a(t)=\left(a_{1}(t), a_{2}(t), \cdots, a_{n_{1}}(t)\right)$

$b(t)=\left(b_{1}(t), b_{2}(t), \cdots, b_{n_{2}}(t)\right)$ symbols are as before. The program is the bi-level linear programming which will be studied that the upper level cost coefficient the linear function of parameter $t$.

In order to guarantee the existence of the optimal solution, we usually have assumption on bi-level programming problem.

Hypothesis H2.1 Admissible set has boundary.

Hypothesis H2.2 When the upper level variable is given, and the lower problem have feasible solutions, the lower problem exists the only optimal solution.

Hypothesis H2.3 When the upper level variable $\bar{x}$ is given, $\bar{y} \in R(\bar{x})$ is the non degenerate vertex of $S(\bar{x})$ and $\bar{y}$ satisfies the strict complementary condition (that is the 
complementary variable of tight constraint is above and beyond zero strictly).

Conclusion 1 The vertices in feasible set GBLP are also the vertices of admissible set.

Conclusion 2 If GBLP has limited optimum value, it will be fulfilled in admissible set.

\section{The Basic Thought Of Sensitivity Analysisi}

In different assumed conditions, the three forms of model $\mathrm{M}$ can guarantee that as to each feasible $x$, the lower level optimization problem has one and only solution, and the optimal solution of bi-level decision problem must reach in the culmination of admissible set. Suppose $m^{i}\left(x^{i}, y^{i}\right)(i=1, \ldots, N)$ are feasible solutions of linear programming problem

$$
\begin{gathered}
\max _{x} f_{1}(x, y)=a^{T} x+b^{T} y \\
\text { s.t. } \quad A x+B y \leq p \\
x \geq 0, y \geq 0
\end{gathered}
$$

On the basic thought of kth-best [6], if the vertex is the optimal solution of bi-level decision problem it will firstly guarantee

$$
\left(x^{i}, y^{i}\right) \in F
$$

Secondly, the value of $f_{1}\left(x^{i}, y^{i}\right)$ must be equal all greater than the upper level objective function of each feasible culmination, that is $f_{1}\left(x^{i}, y^{i}\right) \geq f_{1}\left(m^{1}\right) \geq \ldots \geq f_{1}\left(m^{i-1}\right) \geq f_{1}\left(m^{i+1}\right) \geq \ldots \geq f_{1}\left(m^{N}\right)$

Therefore, if certain changed data is known, it only needs to calculate and test the new data to see whether (1) and (2) are valid. If so, the optimal solution remains unchanged; otherwise, new optimal solution should be solved.

\section{STEPS Of SENSITIVITY ASNASIS}

The paper discusses the bi-level decision problem of lower level objective function including parameter and the upper level without constraints. Because the objective function is linear function of these variables in certain interval and the constraint conditions are linear equation and inequality, the simplex can be used to analyze such kind of problem. Its general steps are:

Step 1 As to the linear programming problem including parameter $t$, let $t=0$, the procedures of [1] can be used to determine upper level optimal solution, the optimal solution of bi-level programming and all vertices respectively.

Step 2 Take all vertices to the lower level parameter programming to verify their possibilities.

Step 3 Using the condition of optimality and feasibility to analyze the variation range when make the optimal solution unchanged.

Step 4 Analyze the change of optimal solution when the parameter goes beyond this range. If the condition of optimality or feasibility doesn't meet the need, the simplex can be used to determine new optimal solution and turn to step 3.
The process continues until all possible intervals $(-\infty,+\infty)$ of parameter $t$ are analyzed.

\section{EXAMPLE SOLVING AND ANALysis}

This paper adopts the thoughts of the best method of K-th to give the arithmetic of solving the culmination of constraint set, and by using Matlab programming to obtain culminating points of constraint set. And the simplex is applied to analyze the programming problem of the lower level objective function including parameter and analyze the state of optimal solution.

Example: Try to analyze the changes of optimal solution of bi-level linear programming in the following lower level objective function which coefficient including parameter $t$

$$
\begin{gathered}
\max _{x} f_{1}=1 / 5 x+y \\
\max _{y} f_{2}=(-1+t) y \\
\quad \begin{array}{c}
\text { s.t. } \quad-x-2 y \leq-10 \\
\quad x-2 y \leq 6
\end{array} \\
2 x-y \leq 21 \quad \\
x+2 y \leq 38 \\
-x+2 y \leq 18 \\
x \geq 0, y \geq 0
\end{gathered}
$$

Step 1 Solving the bi-level linear programming problem when $t=0$. From the procedures, we can get that all vertices of bi-level programming are $(8,1)$,

$(0,5),(12,3),(16,11),(0,9),(10,14)$, and the optimal solution is $(16,11)$.

Step 2 Putting all vertices into the lower level programming to verify their possibilities.

When $x=10$, respond the parameter $t$ to the original lower level's optimal table, table 1 is gotten:

From table 1, we know $y=2$ is the optimal solution of original lower level programming. As $y=2 \neq 14$,so $(10,14) \notin F$.

Step 3 When ${ }^{t}$ changes, if the optimal solution of original lower level programming remains unchanging, $t \leq 1$ is gotten only when $1 / 2 t-1 / 2<0$.

Step 4 When $t>1$, the original optimal solution is not the optimal one, continue to iterate to get the optimal solution. We have table 2:

From table 2 we know that when $t>1,1 / 2 t-1 / 2<0$, so $y=14$ is the optimal solution of lower level programming, that is $(10,14) \in F$ is the feasible solution.

Thus, when $x=10, t>1,(10,14)$ is the feasible solution, when $t \leq 1,(10,14)$ is not the feasible solution.

In a similar way we can inspect and verify: when $t \leq 1,(16,11),(8,1),(12,3)$ are feasible vertices. when $t>1$, $(16,11)$ is the feasible vertices. 
In sum, when $t \leq 1,(16,11),(0,5),(8,1),(12,3)$ are feasible vertices; $t>1,(16,11),(0,9),(10,14)$ are feasible vertices.

Because

$f(10,14)>f(16,11)>f(0,9)>f(12,3)>f(0,5)>f(8,1)$, th us when

$t \leq 1$, the optimal solution $(16,11)$ of bi-level programming is unchanged. When $t>1$, the optimal solution of bi-level changes and the optimal solution is $(10,14)$.To sum up, with the change of parameters, the solutions of this question are as follow table:

\section{SUMMARIES}

From the instances we know that because only the coefficient of lower objective function has parameter, the upper level objective function and constraint conditions are all not changed, thus the passing vertices and the optimal solutions of upper programming are all not changed. While the coefficient of objective function in lower level programming changes continuously which will influence the admissible set, thus the optimal solution may change. As to the bi-level decision problem of lower level objective function's coefficient including parameter ${ }^{t}$, it discusses the change situation of optimal solution when parameter $t$ changes continuously, so as to provide foundation for decision maker making final decision when the market price fluctuates.

\section{REFERENCES}

[1] TAO Yu-jie. The Sensitivity Analysis of Bi-level Programming. Journal of Tong Hua Normal University [J], 2006.4, PP6-9

[2] LI Zhi-hui; TAO Yu-jie. The Sensitivity Analysis of Bi-level Decision Problem (2). Mathematics in Practice and Theory [J], 2006.10, PP167-172

[3] TAO Yu-jie; DUAN Li-fen; CUI Yun-an. The Sensitivity Analysis of Bi-level Decision Problem (3). Journal of He Nan Normal University [J], 2009.4, PP145-147

[4] LIU Hong-ying; LIU San-yang; ZHOU Shui-sheng. Linear Programming of Bi-level Broad Sense. Journal of Systematic Engineering [J], 2000.2, PP131-135

[5] LI Zhi-hui; TENG Chun-xian; LI Lei; LI Hao-bai. Using the Best Solution of K-th to Solve the Problem of Price Control. Journal of Harbin University of Science and Technology[J], 1999.2, PP108-110

[6] TAO Yu-jie. The Sensitivity Analysis of Bi-level Programming [D], 2004, PP22-33

TABLE I. THE CASE OF CHANGE OF THE ORIGINAL LOWER LEVEL PROGRAMMING'S OPTIMAL TAB

\begin{tabular}{|c|c|c|c|c|c|c|}
\hline $\mathbf{b}$ & $\mathbf{y}$ & $\mathbf{y}$ & $\mathbf{y}^{2}$ & $\mathbf{y}^{3}$ & $\mathbf{y}^{4}$ & $\mathbf{y}^{5}$ \\
\hline 4 & 0 & 1 & -1 & 0 & 0 & 0 \\
2 & 1 & 0 & $-1 / 2$ & 0 & 0 & 0 \\
3 & 0 & 0 & $-1 / 2$ & 1 & 0 & 0 \\
24 & 0 & 0 & 1 & 0 & 1 & 0 \\
24 & 0 & 0 & 1 & 0 & 0 & 1 \\
\hline & \multicolumn{7}{|c|}{$-1 / 2 t-1 / 2$} \\
\end{tabular}

TABLE II. THE RELATIVE SIMPLEX TABLE OF LOWER LEVEL PROGRAMMING WHEN $t>1$

\begin{tabular}{|r|c|c|c|c|c|c|}
\hline $\mathbf{b}$ & $\mathbf{y}$ & $\mathbf{y}^{1}$ & $\mathbf{y}^{2}$ & $\mathbf{y}^{3}$ & $\mathbf{y}^{4}$ & $\mathbf{y}^{5}$ \\
\hline 28 & 0 & 1 & 0 & 0 & 0 & 1 \\
14 & 1 & 0 & 0 & 0 & 0 & $1 / 2$ \\
15 & 0 & 0 & 0 & 1 & 0 & $1 / 2$ \\
0 & 0 & 0 & 0 & 0 & 1 & -1 \\
24 & 0 & 0 & 0 & 0 & 1 \\
\hline
\end{tabular}

TABLE III. THE SOLUTION SITUATION OF BI-LEVEL PROGRAMMING PROBLEM

\begin{tabular}{|c|c|c|c|}
\hline$t$ & $\begin{array}{c}\text { The optimal } \\
\text { solution of upper } \\
\text { level }\end{array}$ & $\begin{array}{c}\text { The optimal } \\
\text { solution of original } \\
\text { programming }\end{array}$ & $\begin{array}{c}\text { The optimal } \\
\text { solution of new bi- } \\
\text { level programming }\end{array}$ \\
\hline$-\infty<t \leq 1$ & $(10,14)$ & $(16,11)$ & $(16,11)$ \\
\hline $1<t<+\infty$ & $(10,14)$ & $(16,11)$ & $(10,14)$ \\
\hline
\end{tabular}

